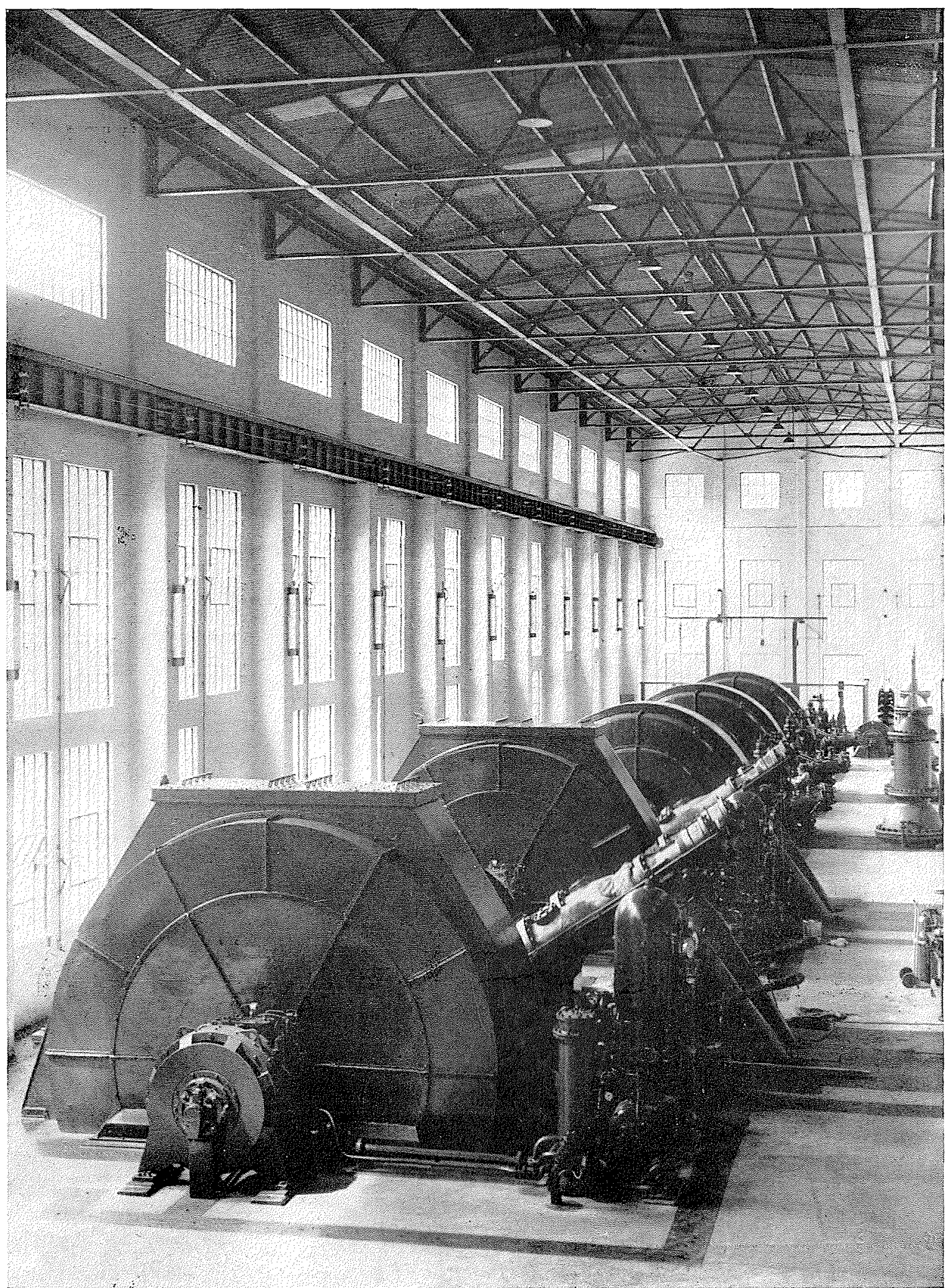

Vue intérieure de la centrale ce Tarralcah en Nouvelle-Zélande. La force totale développée est de l'ordre de $105.000 \mathrm{cv}$ 


\title{
LES ANCIENNES UTILISATIONS DE L'EAU
}

\section{par M. FERRENDIER}

\author{
Docteur en Droit
}

Jusqu'au moment où se répandit l'emploi de la machine à vapeur, au $X \mid X^{*}$ siècle, la force hydraulique a été, avec le vent, le seul élément naturel dont I'homme sut disposer, aussi les utili. sations des roues ont-elles été multiples et variées.

Le mouvement de l'eau une fois reçu sur la roue et régularisé, il était possible d'en tirer parti d'une foule de façons. Les moulins à blé sont connus de tout le monde, mais les roues pouvaient, sans grande modification, transmettre le mouvement à bien d'autres appareils que l'on nommait autrefois des artifices". Elles servaient à battre la pâte à papier, à fouler le drap, les toiles et les pelleteries, à pulvériser des écorces pour le tannage, à presser des noix, scier le bois et les pierres, filer les cocons, mouliner la laine. On les employait dans l'exploitation des mines pour séparer le minerai de sa gangue, dans la métallurgie, pour insuffler l'air dans les fourneaux, pour forger le métal avec des martinets, pour l'étirer ou le laminer. Si le nom de moulin se donnait le plus souvent, comme aujourd'hui, à l'usine dans laquelle se faisait la transformation du blé en farine et issues, c'était aussi le terme général servant à désigner les usines à eau et il était d'usage courant de parler des moulins à papier, à huile, à foulon..

Les contemporains étaient vivement frappés de l'action si efficace des eaux courantes, ainsi qu'en témoigne, entre autres, cette jolie description du XII' siècle, citée par I'Abbé Vacandard et concernant l'abbaye de Clairvaux :

«Admis dans l'abbaye autant que le mur qui fait fonction de portier le permet, le fleuve s'élance d'abord avec impétuosité dans le moulin où il est très affairé et se donne beaucoup de mouvement, tant pour broyer le froment sous le poids des meules, que pour agiter le crible fin qui sépare la farine du son. Le voici déjà dans l'édifice voisin : il remplit la chaudière et s'abandonne au feu qui le cuit pour préparer la boisson des moines, la bière si, par hasard la vigne a donné à l'industrie des vignerons la mauvaise réponse de la stérilité. Mais le fleuve ne se tient pas pour quitte. Les. foulons établis près du moulin l'appellent à eux. Dans'le moulin, il s'est occupé de préparer la nourriture des frères; on est donc en droit d'exiger que, maintenant, il songe à leur habillement. II ne contredit pas et ne refuse rien de ce qu'on lui demande. II élève ou abaisse alternativement ces lourds pilons, ces maillets ou, pour mieux dire, ces pieds de bois des foulons. Quand il a fait tourner d'un tournoiement accéléré, tant de roues rapides, il sort en écumant; on dirait qu'il est moulu lui-même. Au sortir de là, il entre dans la tannerie où, pour préparer les matières nécessaires à la chaussure des frères, il montre autant d'activité que de soin; puis il se partage en une foule de petits bras et va, dans sa course officieuse, visiter les différents services, cherchant diligemment partout ceux qui ont besoin de son ministère, pour quelque objet que ce soit, qu'il s'agisse de cuire, tamiser, tourner, broyer, arroser, laver ou mou. dre, offrant son concours, ne le refusant jamais. 》

L'eau courante semblait avoir ainsi un rôle universel. Cependant quelques applications de la roue hydraulique ont dominé toutes les autres, et de bien loin, en raison de leur utilité sociale primordiale. Parmi elles, figurent les moulins à blé qui fournissaient à l'homme un aliment de première nécessité, les moulins à foulon qui l'aidaient à se vêtir, les moulins à papier qui lui permettaient de répandre sa pensée, et aussi les machines que les roues animaient dans les mines, les forges et les fonderies et qui lui procuraient les matériaux les plus nécessaires à sa vie quotidienne.

Toutes ces industries se trouvaient dans l'étroite dépendance de la force hydraulique. A cette époque, l'énergie électrique ne s'interposait pas, comme aujourd'hui, entre le récepteur hydraulique et la fabrique en permettant de les placer à de grandes distances les uns des autres. Les machines utilisées étaient en connexion directe avec les roues hydrauliques, un même bâtiment les abritait, elles formaient un ensem. ble qui ne pouvait être dissocié.

Les roues étaient l'élément le plus essentiel de ces usines et c'est d'elles que dépendait leur fonctionnement. Aussi, quand on voulait faire entendre qu'une usine allait s'arrêter, disait-on que « I'on mettait la pelle bos». L'empellement de la roue étant fermé, celle-ci s'arrêtait et toute I'usine avec elle.

1 ll est donc intéressant de voir comment s'organisaient les fabrications autour de la roue que le cours d'eau faisait tourner sans se lasser, et c'est 


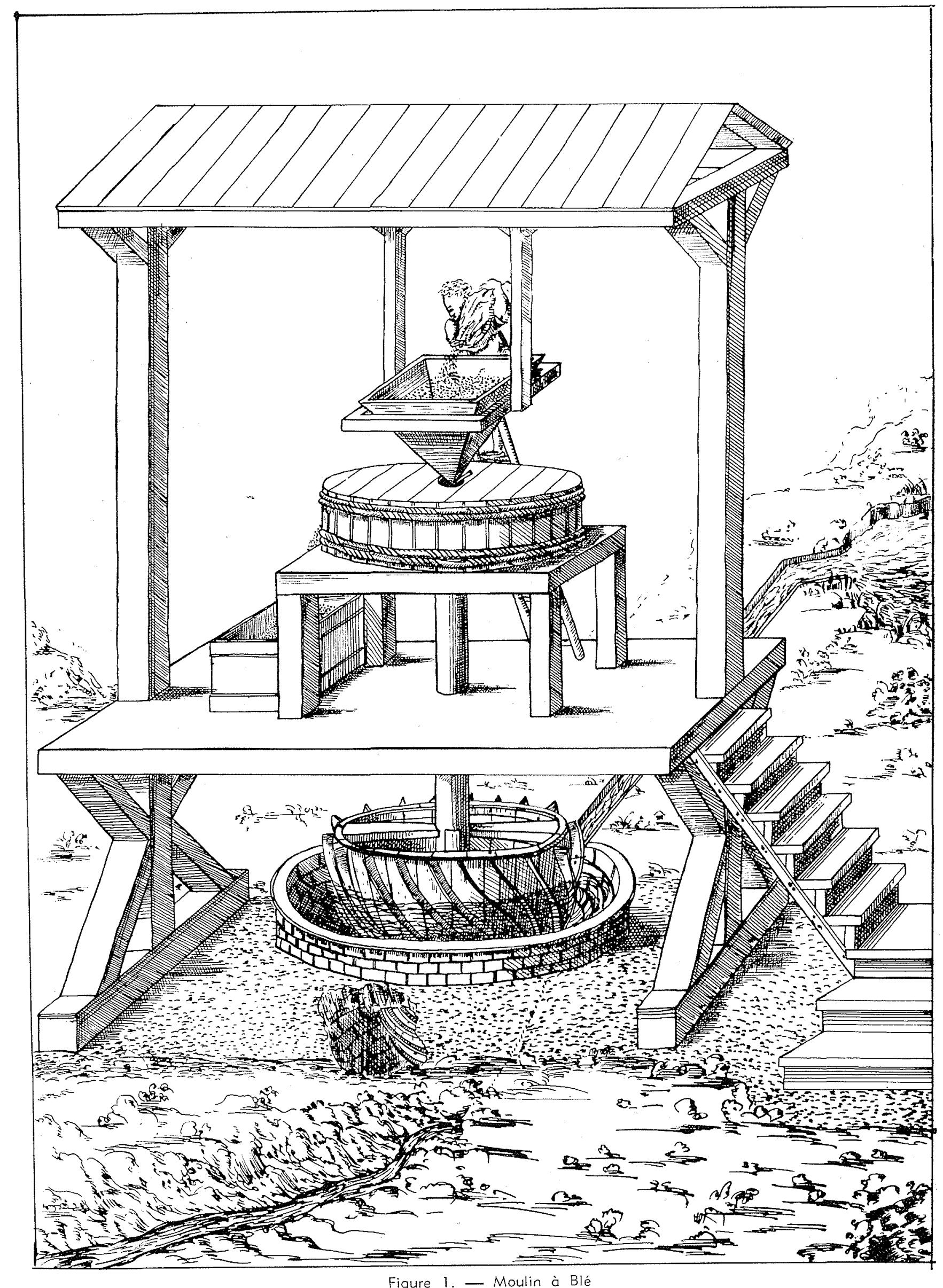




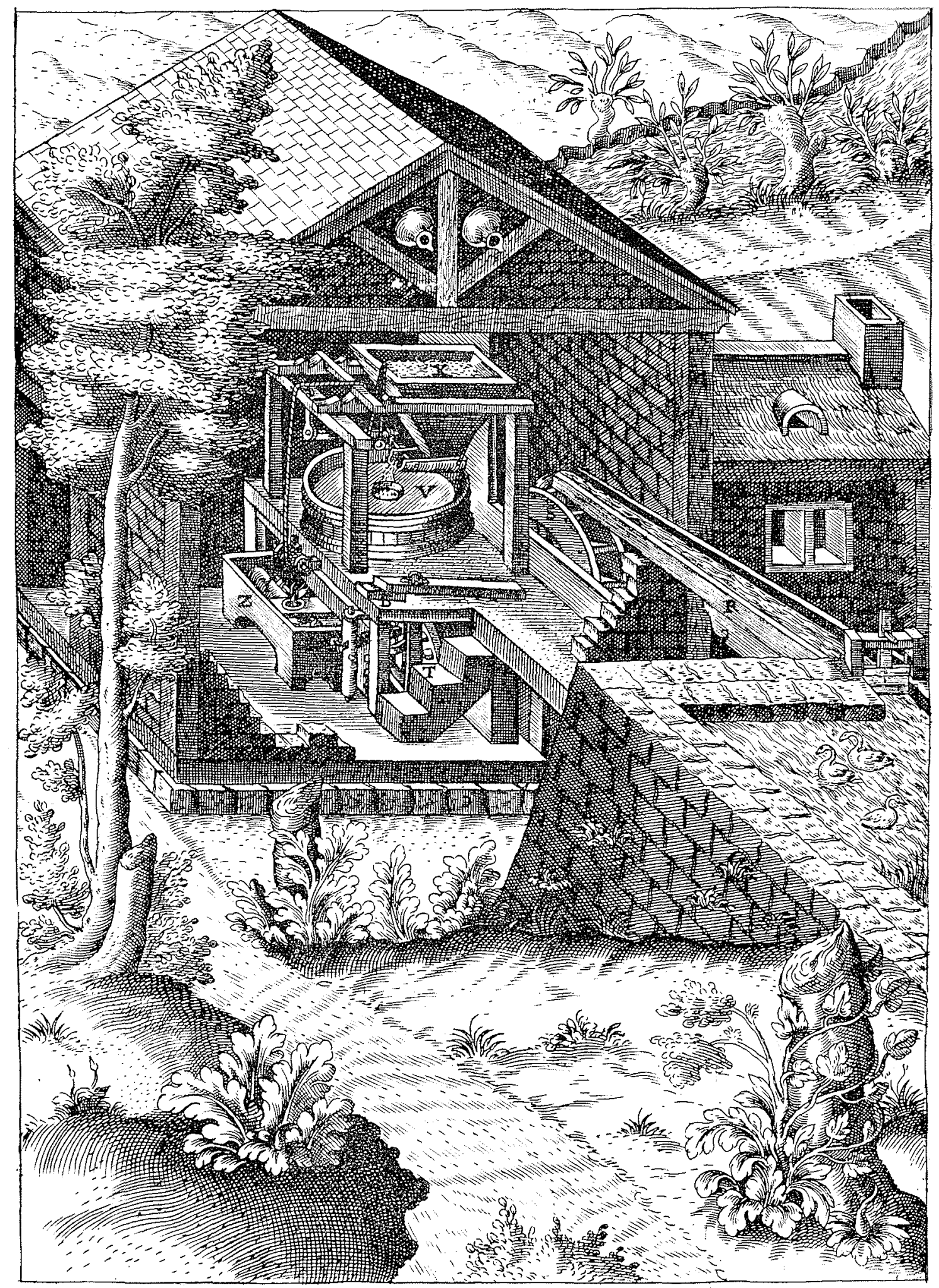

Figure 2. - Ramelli : "Les artificieuses machines " 
ce que nous allons examiner pour les industries qui viennent d'être énumérées.

\section{CHAPITRE}

\section{LES MOULINS A BLE}

Ce sont les plus anciennes usines, les premières que connut le Moyen-Age. Par la suite, elles ont servi de modèle à tous les autres moulins.

Le moulin à blé est destiné à préparer le pre. mier et le plus nécessaire de tous les aliments: son emploi constituait un très grand progrès, car il y a une grande différence entre broyer le grain avec un rouleau ou le piler dans un mortier après l'avoir soumis à une certaine torréfaction, comme on le faisait primitivement et le moudre, le remoudre, en bluter les farines et les gruaux.

Nos ancêtres ont fait usage de mortiers ou de pierres plates creusées au centre par le frottement du pilon, instrument brut et raboteux dont la tête fut ensuite armée de fer et hérissée d'aspérités. On imagina plus tard de broyer les grains entre deux meules, suivant un procédé qui est attribué par Pausanias, dans sa « Description de la Grèce », à un roi de Lacédémone, alors que la tradition antique y voyait un don de Déméter. Deux siècles avant Jésus-Christ, Caton l'Ancien parle du moulin à meules tournantes qu'il dénomme «molae versatiles asinariae ». Strabon, Lucrèce et Vitruve ont fait également mention des moulins à blé, AuluGelle, Ovide et Lucius Apulée ont cité lés moum lins à bras dont on s'est tout d'abord servi.

Le travail de moudre les grains au manège était fort pénible, c'était l'emploi des esclaves et même des criminels. PLAUTE fut réduit, dans sa jeunesse, pour subsister, à tourner la meule d'un moulin de cette espèce.

SEPTIMENIE, nourrice d'un des Fils de CHIL. DEBERT, convaincue de plusieurs crimes, fut condamnée à être fustigée, flétrie d'un fer chaud au visage et reléguée dans un village pour $y$ tourner toujours la meule du moulin.

Un premier perfectionnement consista à faire mouvoir les moulins tournants au moyen d'un manège mû par des animaux : ânes, mulets ou chevaux. L'exclamation joyeuse qui figure sur un graffito célèbre: «Labora Aselle quomodo laboravi » concrétise bien le soulagement avec lequel les esclaves accueillirent ce nouveau mode de propulsion. Mais le moulin à eau devait allé. ger bien plus encore le labeur des hommes ef même celui des femmes, comme en témoigne une épigramme grecque du premier siècle attribuée à Antipatros de Thessalonique : « Ne mettez plus la main au moulin, ô femmes qui tournez la meule, dormez longtemps quoique le chant du coq annonce l'aurore, car CERES a chargé les nymphes du travail qui occupait vos bras. Ce que faisaient vos mains laborieuses, les Naïades le feront. Déjà elles s'élancent jusqu'au haut d'une roue qui fait tourner un essieu. Celui-ci entraine avec violence la masse pesante des meules. Nous voilò revenus à la vie heu. reuse, calme et facile de nos premiers pères: nous $n^{\prime}$ civons plus à nous inquiéter de nos repas, et nous allons jouir enfin des doux présents de Cérès 》.

On trouve dans Vitruve une description du moulin à eau tel à peu près, qu'on l'a connu pendant des siècles: «Sur l'axe horizontal de la roue à aubes se trouve une roue dentée (tympanum dentatuml qui tourne en même temps que la roue (versatur cum rota pariter). Cette roue verticale engrène (continetur) sur une autre disposée horizontalement, plus grande que la première, ce qui donne aux meules un mouvement circulaire $\gg$.

A travers les siècles, de César à Louis XV1, le mécanisme des moulins à blé ne s'est guère modifié. Ils étaient fort répandus, si bien que Bélidor ne craint pas de justifier l'étude qu'il leur consacre : « II peut paraître étrange que je me sois donné la peine d'écrire sur un sujet aussi connu que celui-ci... Les moulins sont communs, à la vérité; c'est justement ce qui en prouve l'utilité et la nécessité de chercher les moyens de les perfectionner $\gg$.

Les moulins qu'a connus l'ancien régime, étaient des ouvrages de charpente. Au dire de MALOUIN, le mécanisme du moulin était une machine aussi composée pour le moins, que celle d'une grande horloge. La roue transmettait le mouvement à un arbre horizontal dit gisant ou tournant, sur lequel était monté le rouet ous hérisson, qui jouait le rôle de roue d'engrenage. Le rouet était formé de quatre pièces de bois dites chanteaux assemblées en carré, dont la surface extérieure était courbe et représentait un quart de cercle. Les chanteaux étaient reliés à 'l'arbre tournant au moyen de quatre pièces en croix : les embrasures ou croisées du rouet. Tout autour du rouet, étaient disposées des chevilles saillantes qui engrenaient sur la lanterne.

Celle-ci faisait office de pignon denté. Elle était formée de deux pièces de bois circulaires oppelées tourtes, placées horizontalement et reliées entre elles par des baguettes de bois dur, les fuseaux, qui étaient accrochés au passage par les chevilles du rouet.

Les chevilles et les fuseaux étaient faits d'un bois dur : poirier sauvage ou cormier.

La lanterne était traversée verticalement par le gros fer à section carrée qui reposait sur un pas ou crapaudine, qui pouvait être relevé ou 


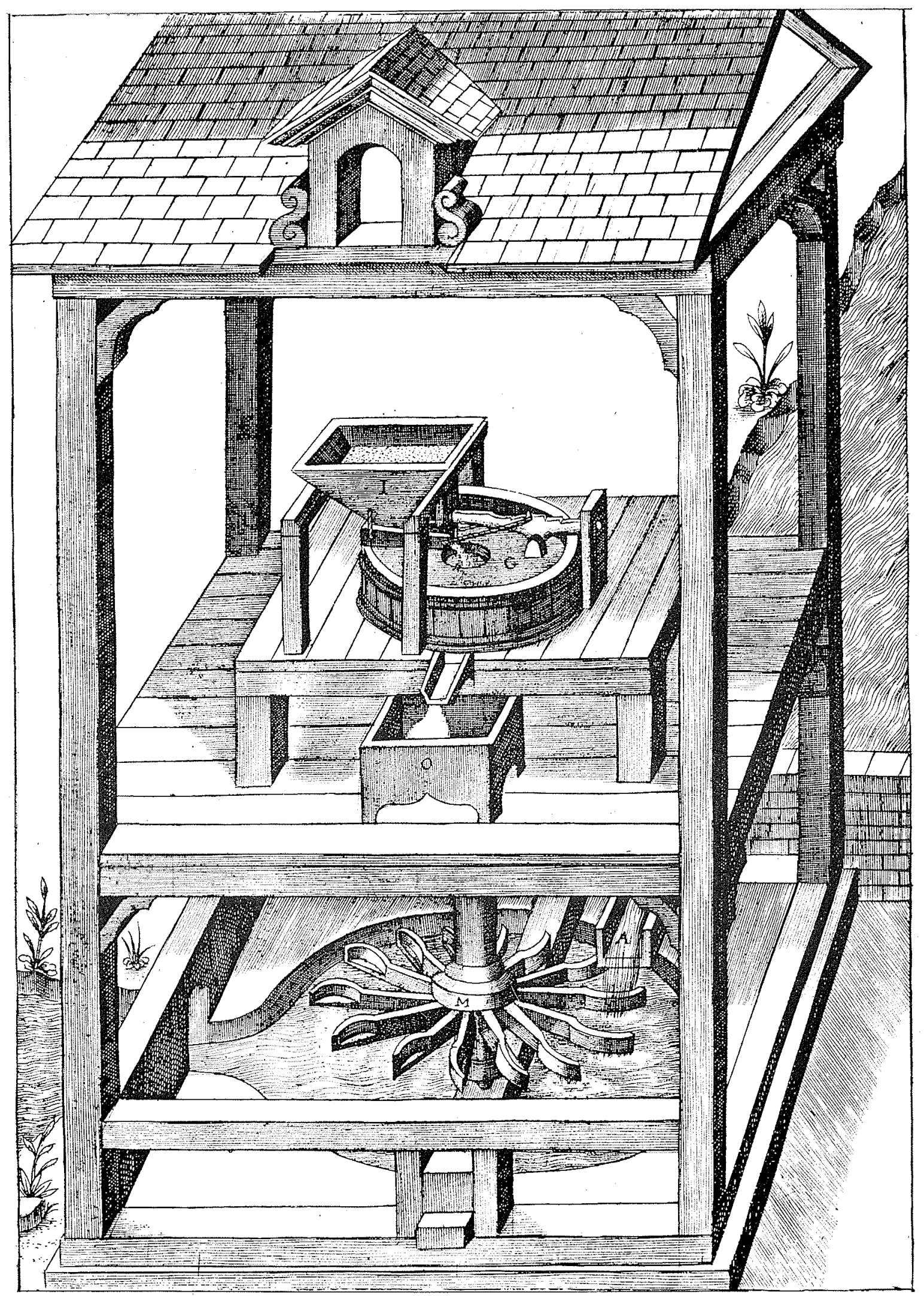

Figure 3. - Ramelli : "Les artificieuses machines » 
abaissé de façon à modifier la distance qui existait entre les meules. Le gros fer était aminci ò sa partie supérieure qui constituait la fusée et se terminait par une pièce de fer à section carrée, le papillon. Il traversait la meule fixe, meule gisante ou dormante, entre des boites de bois qui garnissaient le trou de la meule et empêchaient le grain de passer; il entraînait la meule supérieure dénommée meule tournante, courante ou volante, dans l'œillard de laquelle était scellée une pièce de fer à quatre branches, l'anille, présentant en son milieu un trou carré qui recevait le papillon du gros fer.

Les meules étaient environnées d'un cercle ou caisse, l'enchevêtrure, dans laquelle la meule de dessus faisait tomber le grain moulu. Les planches qui en formaient le pourtour se nommaient archures parce qu'elles étaient courbées en arc; celles qui recouvraient le dessus se nommaient couverceaux.

Les meules, dont le poids était considérable, 1.000 d 1.500 livres chacune, étaient posées sur une forte charpente, nommée beffroi, composée de quatre montants robustes réunis en haut et et bas par des traverses horizontales de fort équarrissage. Sur celles du haut reposait le plancher sur lequel la meule gisante était assujettie.

Les meules étaient faites de pierres dures et

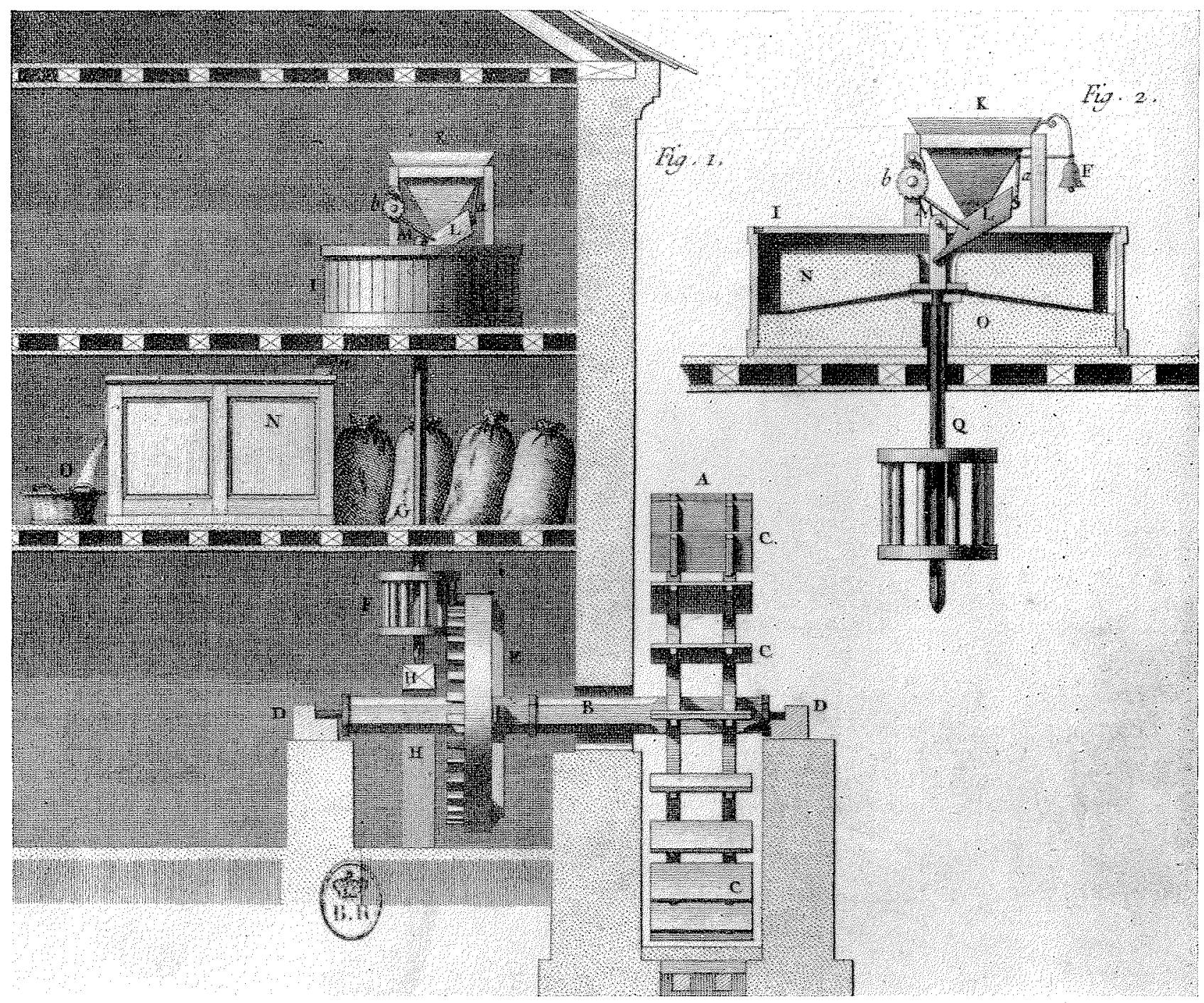

Figure 4. - Abbé Poncelet : « Histoire Naturelle du Froment »

C C - Aubes.

$B$ - Arbre ou grand essieu de bois.

A - Grande roue de 16 pieds de diamètre.

DD - Tourillons de fonte.
E - Rouet de 4 pieds de diamètre.
$F$ - Lanterne.
G - Essieu de fer.
H - Palier.

1 - Tambour.

K - Trémie.

L. - Auget. 
raboteuses, taillées en rond, d'un pied environ d'épaisseur et d'un diamètre plus ou moins grand. On les faisait fréquemment venir de la Brie ou des environs de La Ferté-sous-Jouarre: Rouen était aussi un grand marché de meules. Le grain à moudre était versé dans la trémie, sorte d'auge carrée faite en entonnoir, au fond de laquelle était pratiquée une petite ouverture qui donnait dans un auget animé d'un mouvement de va-et-vient, par l'intermédiaire d'une pièce de bois, le frayon tournant avec l'arbre des. meules. Le grain tombait de l'auget sur la meule par l'ouverture ou cil de la meule courante et il était broyé.

Il y avait dans la plupart des moulins, une petite clochette qui se trouvait reliée par une corde d̀ un poids posé sur le grain; cette clochette se mettait à tinter lorsque il n'y avait presque plus de grain dans la trémie. Le meunier ou le garde-moulin était ainsi averti d'avoir à recharger la trémie, faute dè quoi le mouvement des meules aurait vite fait jaillir des étincelles qui auraient risqué de mettre le feu au moulin. Ainsi, à charge d'avoir l'oreille fine, le meunier pouvait-il s'endormir sans crainte.

Le départ de la farine et du son se pratiquait au moyen de blutoirs ou bluteaux faits d'étamine ou de crin, mus par le babillard, animé lui-même par le mouvement de la lanterne. L'installation pouvait comprendre un second bluteau : le dodinage. Suivant que la farine était ou non séparée du son ou des gruaux, on avait diverses sortes de moutures aux noms pittoresques: la mouture septentrionale, la mouture en grosse ou par économie, la mouture rustique pour le pauvre, rustique pour le bourgeois, la mouture marchande, méridionale, à la lyonnaise.

La plupart des moulins à blé étaient primiti. vement construits en bois; plus tard, ils le furent en pierre. En Normandie, quelques sei. gneurs puissants ou de riches abbayes introduisirent un certain luxe dans ce genre de construction. Parfois on leur donnait une apparence militaire, un aspect de forteresse, comme au moulin de Bourbon-l'Archambault, ou encore une façade de château, comme au moulin de Trévières. Les moulins du Basacle, à Toulouse, furent particulièrement célèbres. Ils comportaient 25 meules de front, entraînées par autant de roues horizontales à cuves. Ils furent consi. dérés comme les plus beaux non seulement du royaume de France, mais de toute l'Europe. Ce qui émerveillait les contemporains, ce n'était pas seulement le grand nombre de meules ran. gées en lignes, mais aussi la hardiesse de la chaussée qui coupait la rivière en biaisant d'un bord à l'autre dans une grande étendue et fai- sait une cascade telle qu'on n'en voyait de pareille nulle part

La faible force développée par les moulins, ainsi que les difficultés de transports obligeaient à les multiplier. L'absence de moyens de communications commodes, rendait utile d'avoir des moulins très voisins des bourgs qu'ils avaient ò desservir. A la fin du XVI1' siècle, VAUBAN en a évalué le nombre au chiffre impressionnant de 60 à 80.000 pour toute la France. Dans certaines villes, il fut longtemps conservé des moulins, dits provisoires, mus à bras d'homme, par des chevaux ou par des ânes, suivant les plus anciens procédés, pour suppléer aux autres en cas de nécessité. II était, en effet, prudent d'avoir de tels moulins dans les villages pour prévenir les famines qui auraient pu survenir, même quand le grain était abondant, lorsque la sécheresse. les inondations ou les gelées paralysaient les moulins à eau.

Une sorte de moulins particulièrement curieuse était constituée par les moulins sur bateaux qui étaient dits moulins pendus ou à nefs. On en fait remonter I'honneur au Général BELISAIRE qui, assiégé dans Rome par les Ostrogoths, et n'ayant plus pour alimenter la ville les moulins de banlieue, en fit installer sur des bateaux retenus par de grosses cordes tendues d'un bord à l'autre du Tibre. On en trouváit sur le Danube et ses affluents, en France sur la Seine, notamment dans la traversée de Paris, la Garonne, le Rhône, la Save et d'autres rivières. Les moulins sur la Garonne qui comptaient parmi les plus anciens remonteraient au X/1 siècle; ic concession en fut accordée par Raymond IV ou par ses prédécesseurs, aux moines du prieuré de la Daurade.

Quant aux moulins du Rhône, ils sont mentionnés dans une bulle du pape Innocent IV, de 1245, où il est question d'un cens de 10 sols à verser par ces moulins au profit du Monastère de St-Pierre. En 1493, il y avait treize moulins sur le Rhône du côté de St-Clair et quatre autres près du pont de la Guillotière. En 1516 on en comptait une vingtaine.

Sur l'Isère, en 1614, les Consuls de la ville de Grenoble autorisèrent $M$. de la Motte-Verdeyer à établir des moulins derrière deux maisons qu'il avait vers le Boeuf, le quartier des tanneurs, de manière qu'en cas de siège, la ville ne soit pas dépourvue de moyens de mouture, comme cela était arrivé en l'an 1590. Quelques mois après, criée fut faite pour annoncer que les moulins de $M$. de la Motte-Verdeyer étaient en état de moudre dans de meilleures conditions que les moulins circonvoisins et à raison de deux livres par charge de blé. Mais ils eurent un sort malheureux car, une quinzaine d'années plus tard, 


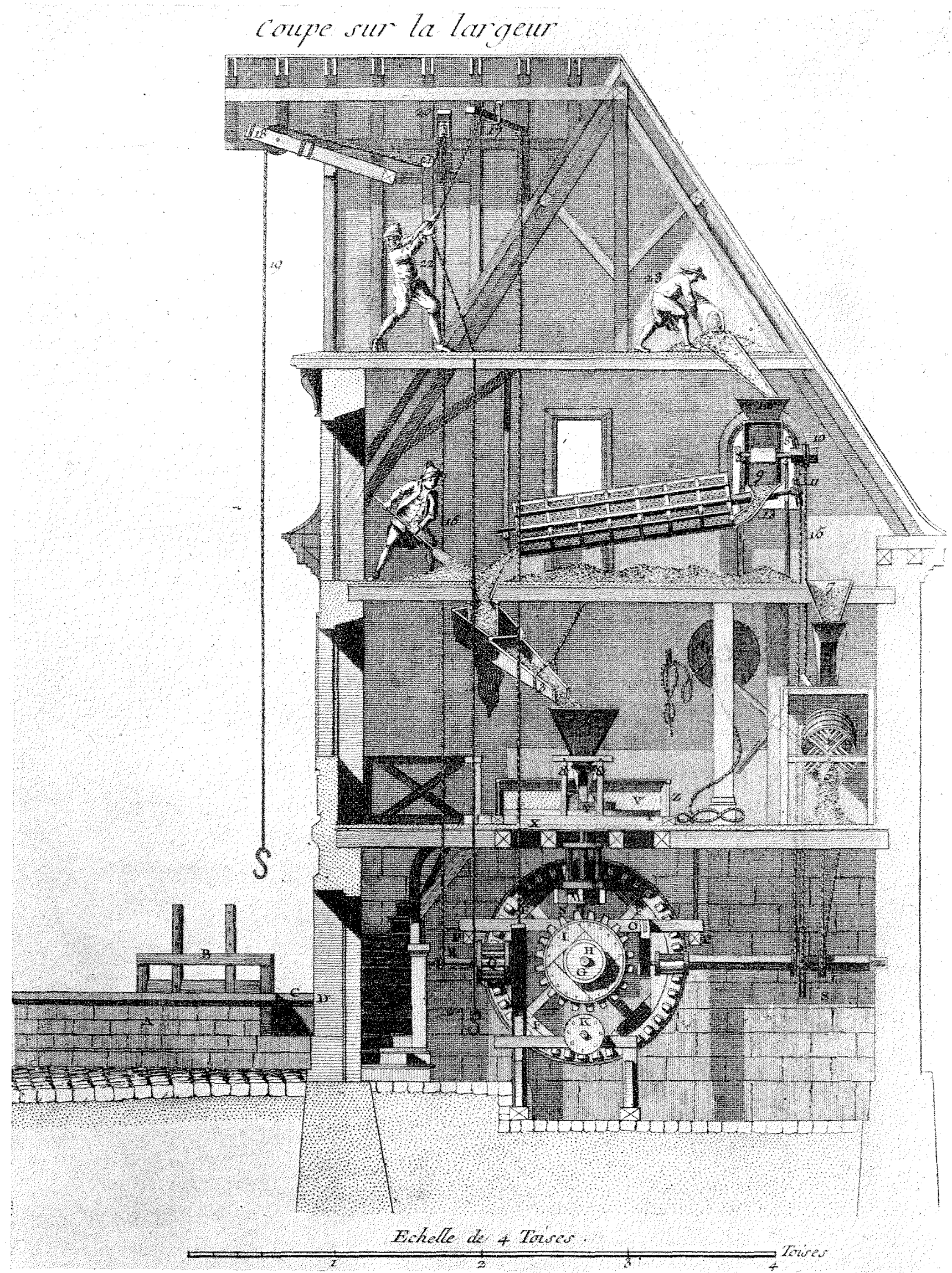

rigure 5. - Suite des Recueils de planches sur les Sciences et les Arts-1777: "Meunier », pl. suppl. 2. 
les bateaux qui les portaient se détachèrent de leurs amarres et vinrent se jeter contre une des piles du pont de bois voisin, qu'ils détruisirent.

Les moulins sur bateaux présentaient des dispositifs divers tant par le nombre des roues que par la manière de les disposer. Ils comportaient fréquemment deux roues, fixées à chaque bout de l'arbre horizontal qui traversait le bateau. Sur le Rhône, on trouvait des moulins pendus, ne comportant qu'une seule roue; d'cutres présentaient un dispositif plus original, constitué par deux bateaux géminés flanqués de deux roues dont l'une était placée entre les deux boteaux qu'elle réunissait; la seconde, appelée roue de fort, se trouvait sur le flane du second bateau, plus petit que le premier. Tous les engre. nages et la charpente étaient en bois. Les deux paires de meules étaient placées sur le grand bateau, ainsi que les magasins, le plus petit bateau ne servait que comme porteur de la se. conde roue.
Ces moulins avaient l'inconvénient d'empiéter considérablement sur le fleuve et de gêner beaucoup la navigation. Ils brisaient le cours de l'eau si bien que les sables et les graviers s'accumulaient dans leur voisinage, en rétrécissant le lit de la rivière. Les tourbillons et l'appel d'eau créés par les bateaux porteurs, entre lesquels tournait une énorme roue, attiraient les bateaux et constituaient un danger permanent En 1736, une barque faisant le service de Lyon, fit naufrage entre des moulins situés à la Quarantaine et 34 des promeneurs furent noyés. A maintes reprises, le déplacement des moulins fut exigé, dans l'espoir qu'il serait possible de leur trouver des emplacements moins gênants.

En 1741, un inventeur du nom de DUBOST essaya un nouveau dispositif n'employant qu'un seul bateau avec une roue située par derrière dans son axe. Ces bateaux furent, à cause de cela, surnommés moulins à queue ou moulins ì comètes. Ils encombraient beaucoup moins le

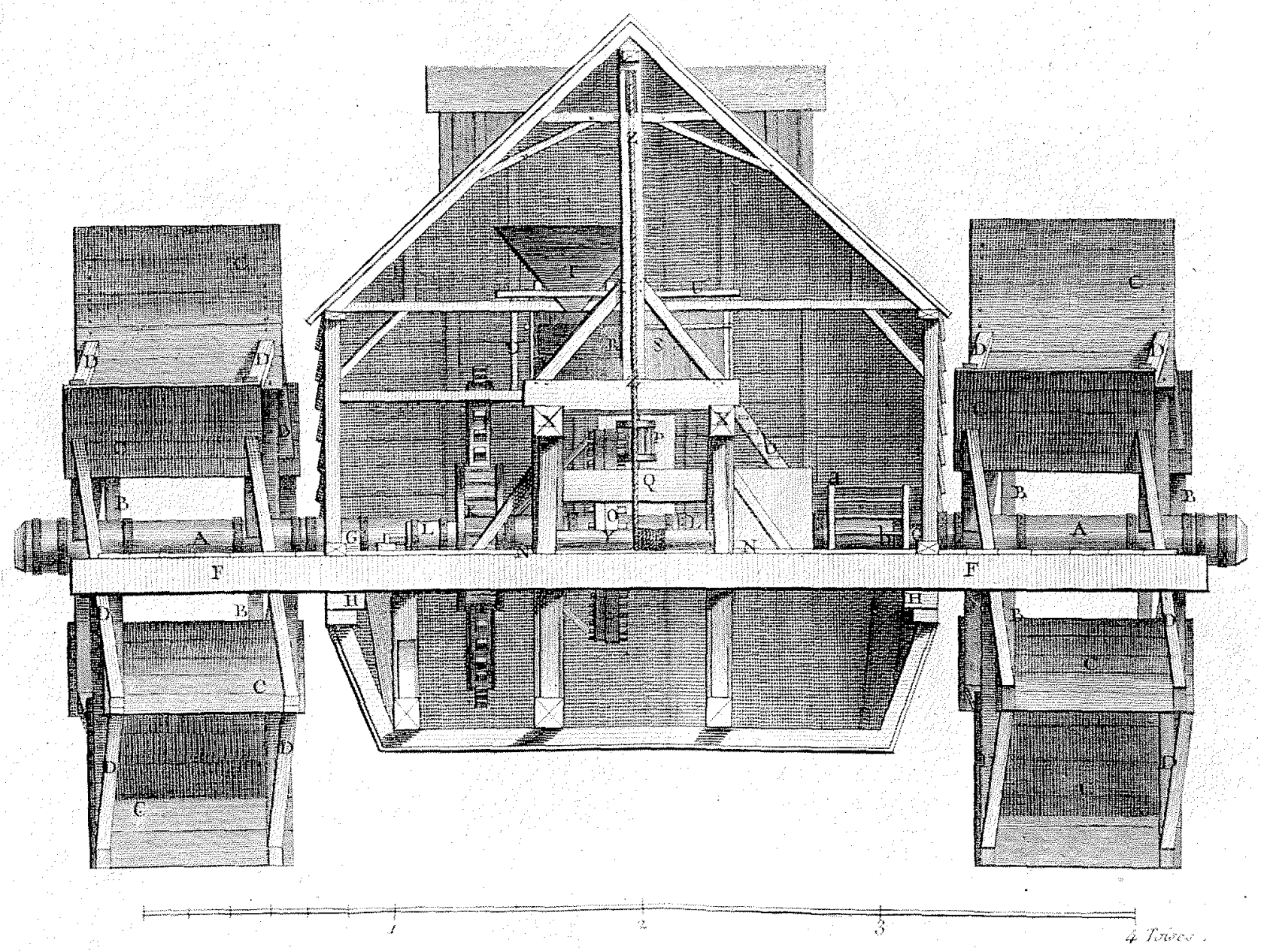

Figure 6. - Encyclopédie. "Moulin à eau », pl. XXXIII 
cours du fleuve et pouvaient être amarrés plus près des berges. Comme maint inventeur, DUBOST eut beaucoup de peine à faire adopter ses idées et $c^{\prime}$ est seulement en 1749 , soit 8 ans après ses premiers essais sur un modèle de petite dimension, que des moulins furent cons. truits d'après ses données.

Cependant, les moulins étaient toujours critiqués et en 1763, I'Académie de Lyon mit au concours, la question suivante : «Déterminer quelle est, sur le fleuve, la construction de moulins la plus avantageuse pour le produit et la moins nuisible à la navigation ». Les Consuls de Lyon se montraient, de leur côté, hostiles à ces moulins et ils auraient voulu provoquer la construction d'un moulin sur terre susceptible de se substituer aux moulins sur bateaux. Malgré cela, les moulins subsistèrent et en 1817, on en comptait encore 27 sur le Rhône. En 1833, de nouvelles contestations surgirent dont les mou. lins triomphèrent encore; ;ils devaient cependant disparaître un à un, avant la fin du siècle.
En dehors des inconvénients que nous avons déjà signalés, et qui étaient de porter tort à la navigation, les moulins à nefs avaient, plus encore que les autres, le désavantage de ne pouvoir moudre quand l'eau était gelée et d'être menacés par la débâcle des glaces. C'est ainsi qu'en 1694, la Saône qui était entièrement prise ayant crû subitement par la fonte des neiges, la débâcle se déclara en un instant : les eaux se répandirent dans la campagne et les glaces entraînèrent, avec les deux ponts de bois de Lyon, les moulins, les radeaux et les bateaux. On peut citer également l'inondation de Montauban de 1764 qui causa des dégâts considérables aux moulins à foulon installés sur la Garonne. Les moulins sur bateaux n'étaient point stables, et penchaient tantôt d'un côté, tantôt de l'autre, alternativement, si bien que les meules n'étaient jamais d'aplomb. Leur seul avantage était de pouvoir moudre quelle que fût la hauteur des eaux.

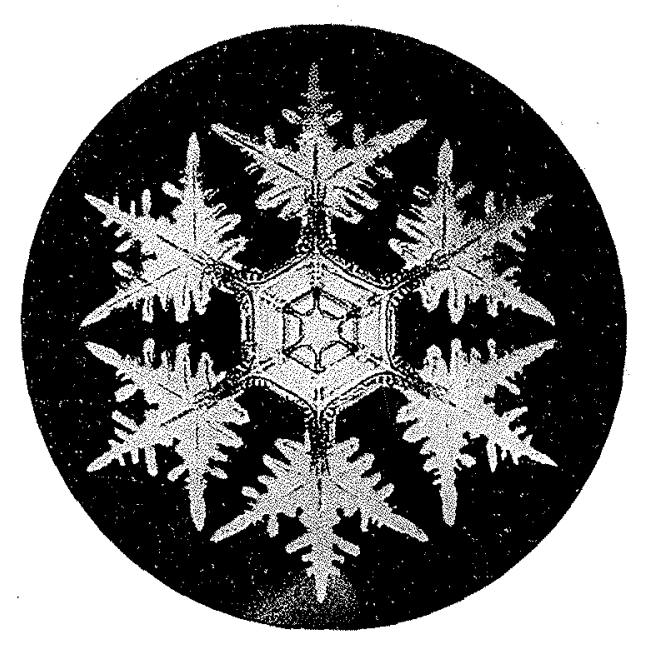

